\title{
Saturation oxygenation pressure index: a non-invasive bedside measure for severity of respiratory disease in neonates on CPAP
}

\author{
Deepti Thandaveshwara ${ }^{1}$ - Ashok Huduguru Chandrashekar Reddy ${ }^{1}$ - Manjunath Vaddamabal Gopalakrishna ${ }^{1}$. \\ Srinivasa Murthy Doreswamy ${ }^{1}$
}

Received: 24 August 2020 / Revised: 30 October 2020 / Accepted: 11 November 2020 / Published online: 20 November 2020

(C) Springer-Verlag GmbH Germany, part of Springer Nature 2020

\begin{abstract}
The treatment of respiratory distress in neonates ranges from non-invasive continuous positive airway pressure (CPAP) to advanced invasive mechanical ventilation. Monitoring on CPAP is often done by calculating oxygenation after blood gas analysis which is an invasive procedure. Saturation oxygen pressure index (SOPI) is a simple, non-invasive, bedside tool to monitor the severity of respiratory illness in neonates on CPAP. This study was aimed at validation of SOP index against AaDO2 and determine the cut-off values of SOPI for A-aDO2 of 70, 85 and100. This was a prospective observational study on 126 neonates with respiratory distress and treated with CPAP. The correlation between SOPI and A-aDO2 was validated using Bootstrap method. There was a significant positive correlation between SOPI and A-aDO2 with the Spearman correlation coefficient (rho) being $0.815(p=0)$. The mean coefficient of correlation after Bootstrap was $0.827(p$ value $<0.0001)$. SOPI values of $1.52,1.57$ and 1.6 predicted $\mathrm{A}-\mathrm{aDO} 2$ value of 70,85 and 100 with a sensitivity of above $80 \%$ and specificity above $90 \%$, respectively.

Conclusion: SOPI has a significant positive correlation of $82.7 \%$ against $\mathrm{A}-\mathrm{aDO} 2$ and can be a valuable tool to assess respiratory distress in neonates without arterial blood gas.
\end{abstract}

What is Known:

- Saturation oxygen pressure index (SOPI) is a non-invasive monitoring tool for neonates on N-CPAP.

- SOPI has a good correlation with AaDO2.

What is New:

- Correlation of SOPI with AaDO2 is now validated.

- Values between 1.52 and 1.88 indicate clinically useful range of AaDO2 for escalation of respiratory support from CPAP.

Keywords SOP index $\cdot$ Non-invasive assessment $\cdot$ Respiratory distress $\cdot$ Neonates

Communicated by Daniele De Luca

Srinivasa Murthy Doreswamy

drdsrinivasa@gmail.com

Deepti Thandaveshwara

deeptiadarsh@gmail.com

Ashok Huduguru Chandrashekar Reddy

drashokhe@gmail.com

Manjunath Vaddamabal Gopalakrishna

vghunsur@rediffmail.com

1 Department of Pediatrics, JSS Medical College, JSS Academy of Higher Education and Research, Mysuru, 70, PRAKRUTHI, BEML 2nd Stage, Rajarajeshwari Nagara, Mysuru, Karnataka, India

\begin{tabular}{ll}
\multicolumn{2}{l}{ Abbreviations } \\
N-CPAP & Nasal continuous positive airway pressure \\
$\mathrm{OI}$ & Oxygenation index \\
$\mathrm{SOPI}$ & Saturation oxygen pressure index \\
$\mathrm{AaDO} 2$ & Alveolar arterial oxygen difference \\
$\mathrm{CDP}$ & Continuous distending pressure \\
$\mathrm{FiO}_{2}$ & Fraction of inspired oxygen \\
$\mathrm{NICU}$ & Neonatal intensive care unit \\
$\mathrm{SpO}_{2}$ & Arterial oxygen saturation \\
$\mathrm{H}_{2} \mathrm{O}$ & Water \\
$\mathrm{PEEP}$ & Positive end-expiratory pressure \\
$\mathrm{ROC}$ & Receiver operative characteristics \\
$\mathrm{IQR}$ & Interquartile range \\
$\mathrm{SD}$ & Standard deviation
\end{tabular}




\section{Introduction}

Majority of the neonates with respiratory distress suffer from restrictive lung pathology which is managed by alveolar recruitment and preventing atelectasis. This is achieved by providing continuous distending pressure (CDP) to the airway via nasal continuous positive airway pressure (N-CPAP) [1]. As the underlying pathology is dynamic, the clinician has to monitor these neonates closely and adjust the pressures or oxygen ( $\mathrm{FiO} 2)$ provided to these babies. Important bedside monitoring parameters are the severity of respiratory distress on clinical examination, which is subjective and $\mathrm{SpO} 2$ [2]. Invasive blood gas analysis and estimation of alveolar-arterial oxygen difference $(\mathrm{A}-\mathrm{aDO} 2)$ are done on an intermittent basis to assess the progress of the disease and make necessary clinical decisions [3].

Many of the practices in the neonatal intensive care are unit specific and hence lack uniformity across the globe $[2,4]$. One such practice is initial respiratory support $[5,6]$. Escalation and de-escalation of the N-CPAP support to the babies with changing severity of the disease in terms of the sequence of altering the $\mathrm{FiO} 2$ and pressure vary among the units and individuals. This highlights the need for having an objective and non-invasive near-continuous assessment tool which can help in making an appropriate clinical decision. One such promising tool is the saturation oxygen pressure index (SOPI).

This incorporates the measure of respiratory support given to the neonate in terms of both $\mathrm{FiO} 2$ and distending pressure along with the measure of response to the support as $\mathrm{SpO} 2$, which is expressed as a number without unit. Initial development of this tool as a concept [7] and its subsequent clinical study [8] for utility in neonates on CPAP has shown a high correlation with arterial alveolar oxygen difference (AaDO2).

Apart from serving as a useful bedside tool, SOPI also brings about uniformity in the expression of the severity of illness and hence useful in research as well. It also reduces the need for invasive blood gas measurement and its implication on workload and financial burden. We validated the correlation of SOPI against $\mathrm{AaDO} 2$.

\section{Objectives}

\section{Primary objective}

To Validate the correlation of SOPI against A-aDO2.

\section{Secondary objective}

To determine the cut-off value of SOPI which represents AaDo2 values of 70,85 and 100 .

\section{Methods}

\section{Study design}

This was a prospective observational analytical study conducted over a period of 12 months in a tertiary neonatal unit.

\section{Subjects}

All neonates admitted to NICU and requiring N-CPAP support were recruited. Neonates who progressed quickly within half an hour for mechanical ventilation were excluded.

\section{Sample size}

SOPI value of 1.6 has $80 \%$ sensitivity for representing $\mathrm{AaDO} 2$ of 70 [8]. Considering this value and assuming absolute precision of $10 \%$ and $95 \%$ confidence limits, we needed to recruit 61 subjects. Validation studies need two sets of samples, one for training and one for testing. We planned to have an equal number in both the sets, and hence, this number was doubled to 122 .

\section{Study}

Neonates admitted in NICU with respiratory distress were managed according to the unit protocol. Babies who were included in the study were put on N-CPAP with either bubble CPAP (Fischer \& Paykel MR850AEU New Zealand) or ventilator CPAP (Macquet Servo i Sweden). Initial pressures were set to $5 \mathrm{~cm}$ of $\mathrm{H} 2 \mathrm{O}$ and $\mathrm{FiO} 2$ at $30 \%$. We do not use a chin strap or any other methods to actively close the mouth of the baby. Continuous SpO2 monitoring was done using pulse oximetry (Philips Sure Sign VM4, China) with the probe placed on the right hand of the baby. Based on the clinical condition and $\mathrm{SpO} 2, \mathrm{FiO} 2$ was adjusted with increments of $5 \%$ and CPAP pressures with increments of $1 \mathrm{~cm}$ of $\mathrm{H} 2 \mathrm{O}$. Target $\mathrm{SpO} 2$ for preterm babies was between 90 and $94 \%$. Maximum tolerated $\mathrm{FiO} 2$ was $40 \%$ and CPAP pressure of $7 \mathrm{~cm}$ of $\mathrm{H} 2 \mathrm{O}$, before proceeding to intubation and mechanical ventilation. Blood gas analysis was done with right radial arterial puncture within 30 min of N-CPAP initiation as per our unit protocol. If we failed to sample the right radial artery, the left radial artery was punctured to collect blood. If the baby received a $\mathrm{FiO} 2$ of $>21 \%$ and the $\mathrm{SpO} 2$ was above $98 \%$, sampling was delayed until $\mathrm{FiO} 2$ was decreased to $21 \%$ or $\mathrm{SpO} 2$ was consistently less than $98 \%$. Further blood gases were done as per the clinical indication. FiO2, CPAP pressure, and $\mathrm{SpO} 2$ were noted prior to obtaining blood gas. $\mathrm{SpO} 2$ was recorded when the baby was calm, and good pleth waves were seen on the pulse oxymeter. Airway suctioning was avoided at least 10 min before obtaining the $\mathrm{SpO} 2$ values and blood gas sample. A single SOPI and its associated $\mathrm{AaDO} 2$ value per 
subject were used for this study. Demographic and other required details were recorded.

SOPI is calculated using the formula SOPI $=(\mathrm{CPAP}$ pressure or PEEP $\times$ FiO2)/SpO2.

$\mathrm{FiO} 2$ and $\mathrm{SpO} 2$ are expressed in decimals. PEEP is positive end-expiratory pressure set on a ventilator when N-CPAP was delivered by the ventilator.

\section{Statistical analysis}

Statistical analysis was done using Microsoft Excel (Office 365). Qualitative variables are summarized as proportions. Quantitative data is summarized as mean and standard deviation or median and interquartile range depending on the distribution. The correlation between SOPI and $\mathrm{A}-\mathrm{aDO} 2$ is calculated as the Spearman correlation (rho). Receiver operative characteristics (ROC) curves were generated using ROC analysis (web-based calculator for ROC curves available at www. jrocfit.org).

In one of the studies analysing the factors associated with extubation failure [9], the mean (standard deviation) $\mathrm{AaDO} 2$ value of babies who failed extubation was found to be 51 (17). This suggested the need for continuing invasive respiratory support in those babies. With this background, Krishnegowda et al. [8] studied the predictive ability of SOPI for mean plus one standard deviation of the above value (rounded off to 70). We had two more additional cut-offs at 2 and 3 standard deviation added to mean (rounded off). Hence, we considered analysing the predictive ability of SOPI for $\mathrm{AaDO} 2$ of 70, 85 and 100. The cut-off value of SOPI for A$\mathrm{aDO} 2$ of 70,85 and 100 was calculated using the ROC curve. Validation of the correlation was done using the Bootstrap method. Bootstrapping was done using real stats pack for Microsoft Excel.

\section{Results}

One hundred and twenty-six babies were recruited. Eleven of them were not included in the analysis as the lab reported air contamination of samples sent for blood gas analysis. One hundred and fifteen babies remained for final analysis.

Eighty per cent of babies were less than $24 \mathrm{~h}$ old and $69.6 \%$ were males. The gestational age of the babies ranged from 28 to 42 weeks. Birth weight ranged from 1000 to $4180 \mathrm{~g}$. Baseline characteristics are depicted in Table 1. The commonest indication for initiation of N-CPAP was respiratory distress syndrome $(69.6 \%)$ followed by congenital pneumonia (9.6\%). Transient tachypnoea of newborn was diagnosed in $8.7 \%$, and meconium aspiration syndrome in $7 \%$. Persistent pulmonary hypertension, patent ductus arteriosus, and apnoea were the indication for N-CPAP in $1.7 \%$ of our babies.
Table 1 Baseline characteristics

\begin{tabular}{ll}
\hline Quantitative Parameter & Median (IQR) \\
\hline Gestation & $35(32-37)$ \\
Birth weight (preterm) & $1650(1340-2260)$ \\
Birth weight (term) & $2850(2670-3230)$ \\
Age at admission (hours) & $22(3-24)$ \\
Duration of CPAP & $1(1-2)$ \\
Qualitative parameter & $N(\%)$ \\
Gender-males, females & $80(69.6), 35(30.4)$ \\
Maturity-preterm, term & $79(68.7), 36(31.3)$ \\
N-CPAP-success, failure & $76(66.1), 39(33.9)$ \\
\hline
\end{tabular}

Sixty-six per cent of the babies were successfully treated with N-CPAP alone. The median and interquartile range (IQR) SOPI in this group was $1.3(1.11-1.52)$. Median (IQR) AaDO2 in this group was 57.35 (32.8-78.5). Of $33.9 \%$ of babies who progressed to receive mechanical ventilation, the median (IQR) SOPI and AaDO2 at the time of intubation were $1.88(1.5-2.25)$ and 108.1 (74.88-136.3), respectively.

Among the babies who were successfully treated with NCPAP alone, $87 \%$ received N-CPAP support for $72 \mathrm{~h}$ or less and rest for a longer duration. Six per cent of the babies needed N-CPAP support for more than 5 days. The longest duration of CPAP given was 9 days for one neonate.

There is a positive correlation between (Fig. 1) SOPI and A$\mathrm{aDO} 2$ with the Spearman correlation coefficient (rho) of $81.5 \%$ $(p=0)$, and $95 \%$ confidence interval is $72.2-93.3 \%$. The

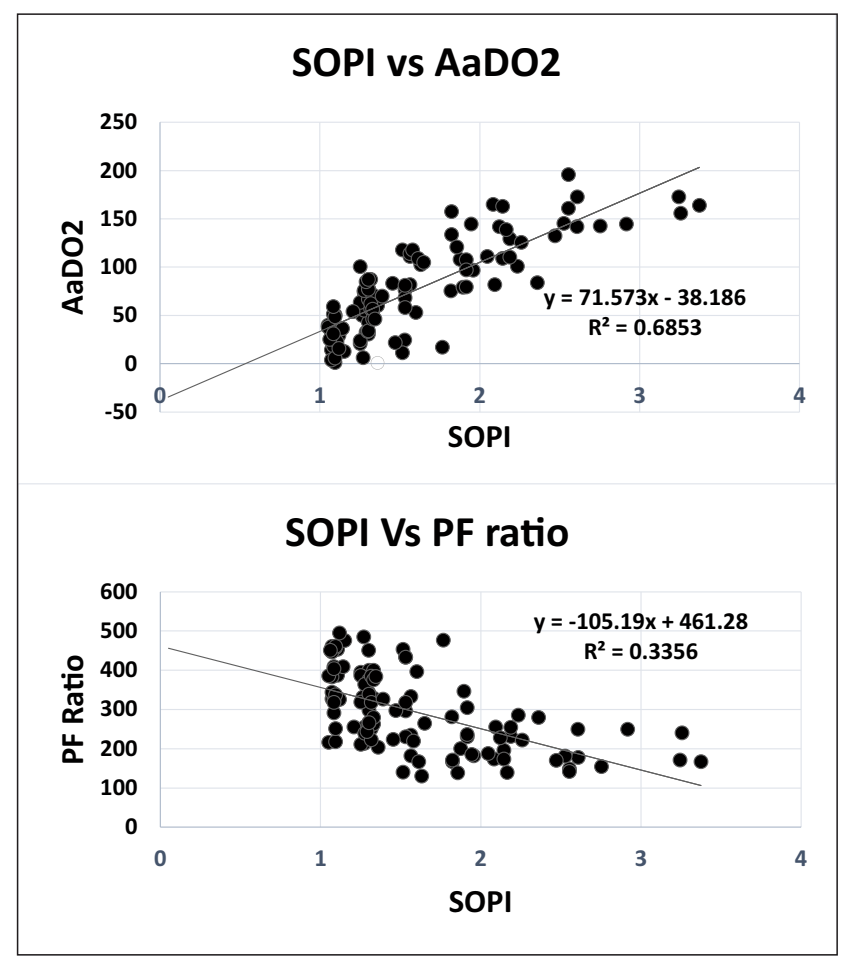

Fig. 1 Correlation between SOPI and AaDO2 
correlation coefficient between SOPI and PF ratio is $57.9 \%$ in the negative direction (Fig. 1). The validation of the correlation between SOPI and $\mathrm{A}-\mathrm{aDO} 2$ was done using the Bootstrap method. The mean coefficient of correlation is $82.7 \%$ with a standard deviation of $2.4 \%$ ( $p$ value of $<0.0001$ ) and $95 \%$ confidence interval of $77.3-87.0 \%$ (Table 2).

ROC curves are generated for three different cut-off $\mathrm{AaDO} 2$ values (Fig. 2). SOPI value of 1.52 had a sensitivity of $80 \%$ and specificity of $92 \%$ for $\mathrm{AaDO} 2$ of 70 . SOPI value of 1.57 had a sensitivity of $81 \%$ and specificity of $90 \%$ of $\mathrm{AaDO} 2$ of 85 , and a value of 1.6 had a sensitivity of $83 \%$ and specificity of $90 \%$ for $\mathrm{AaDO} 2$ of 100. In this era of thrust on non-invasive ventilation, clinicians need more specific indicators to escalate the respiratory support to intubation and ventilation. Hence, we calculated SOPI values which had higher specificity of $95 \%$ for $\mathrm{AaDO}$. SOPI values of $1.55,1.82$ and 1.88 represented $\mathrm{AaDO} 2$ of 70, 85 and 100 , respectively.

Ninety-one per cent of our subjects eventually improved and got discharged. The mortality rate was $9 \%$.

\section{Discussion}

Assessment of the severity of respiratory distress has been an important clinical tool for managing patients of all ages. Various objective measurements for assessment of the severity of pulmonary disease and its effect on oxygenation have been in vogue. Commonly used parameters are $\mathrm{PaO} 2$ to $\mathrm{FiO} 2$ ratio ( $\mathrm{PF}$ ratio), alveolar arteriolar oxygen difference (AaDO2), and oxygenation index (OI). PF ratio is well studied in both adults in older children. It can be used on children even on minimal respiratory support like oxygen supplementation. These parameters for objective assessment in adults were well defined using $\mathrm{PaO} 2$ : $\mathrm{FiO} 2$ and $\mathrm{SaO} 2$ : $\mathrm{FiO} 2$ [10]. The same parameters were shown to be useful in children too [11].

The oxygenation index, on the other hand, gives information on the oxygenation status of the patients who are on mechanical ventilation. This parameter is well studied in neonates as well. This cannot be used if the babies are not on mechanical ventilator support.

Table 2 SOPI validation using the Bootstrap method

\begin{tabular}{ll}
\hline Before bootstrap & \\
Correlation & $0.837(83.7 \%)$ \\
Standard error & $0.053(5.3 \%)$ \\
$95 \%$ confidence interval & $0.722-0.932(72.2-93.2 \%)$ \\
After bootstrap & \\
Mean correlation & $0.827(82.7 \%)$ \\
Standard deviation & $0.024(2.4 \%)$ \\
$95 \%$ confidence interval & $0.773-0.870(77.3-87 \%)$ \\
P value & 0.000 \\
\hline
\end{tabular}

$\mathrm{AaDO} 2$ is a much more comprehensive measurement that can be used in both neonates and older children as well. AaDO2 measurement needs calculation of both alveolar oxygen and arterial oxygen which is influenced by the presence of carbon dioxide as well. In other words, $\mathrm{AaDO} 2$ comprehensively determines the result of the interaction between respiratory pathology and the effect of treatment the patient is receiving.

However, all these parameters need a measurement of arterial blood gas.

SOPI was studied primarily in neonates to assess the severity of respiratory illness. Our study shows an excellent correlation between SOPI and AaDO2. SOPI had a good correlation with the PF ratio as well. Due to the inherent factors associated with foetal haemoglobin, PF ratio alone is not as useful in neonates as in older children.

$\mathrm{N}$-CPAP is one of the highly successful methods of treating neonates with respiratory distress. However, the success depends on close clinical monitoring and revisiting the management strategy frequently as the pathology progress.

A-aDO2 is a measure of the severity of the pulmonary disease. A-aDO2 is normally $<10$ in healthy adults. As neonates have higher physiological dead space, it can be as high as 25 [10]. AaDO2 estimation requires blood gas measurement [10].

Currently, there is no prescribed cut-off $\mathrm{AaDO} 2$ for advancing the respiratory support from CPAP to mechanical ventilation in babies with progressive restrictive lung pathology. This led us to extrapolate the data provided in a retrospective study by Hiremath et al. [9]. In this study, the mean (SD) pre-extubation $\mathrm{AaDO} 2$ in babies who failed extubation was 51 (17). This suggested the need for continued mechanical ventilation. Rephrasing from the perspective of this study, this value suggests that CPAP support will be insufficient to support the respiration in these babies. Hence, $\mathrm{AaDO} 2$ value of 70 (rounded off from $51+17=68$ ) was considered as indicative of a need for escalation to mechanical ventilation.

Blood gas analysis is invasive, requiring an indwelling arterial catheter or frequent arterial/capillary punctures. A significant failure rate of 7.9 to $8.9 \%$ in obtaining an adequate sample is reported [11]. A serious complication of limb ischemia associated with repeated arterial punctures ranges from 1.5 to $35 \%[12,13]$. Further blood gas analysis requires technical expertise and is expensive. This underlines the need for a non-invasive bedside tool to overcome these challenges.

Oxygen saturation index $[14,15]$ is one such non-invasive tool that is used to objectively assess the severity of respiratory distress in neonates. However, its utility is limited only to neonates on mechanical ventilation.

Respiratory severity score is another non-invasive tool available for neonates on mechanical ventilation [16]. This score can be obtained by escalating the respiratory support to achieve a target $\mathrm{SpO} 2$ of $93 \%$. This means that the calculation cannot be done on a real-time basis as one has to administer treatment to estimate the score. It is non-intuitive as 
Fig. 2 ROC curve for various $\mathrm{AaDO} 2$ cut-off values

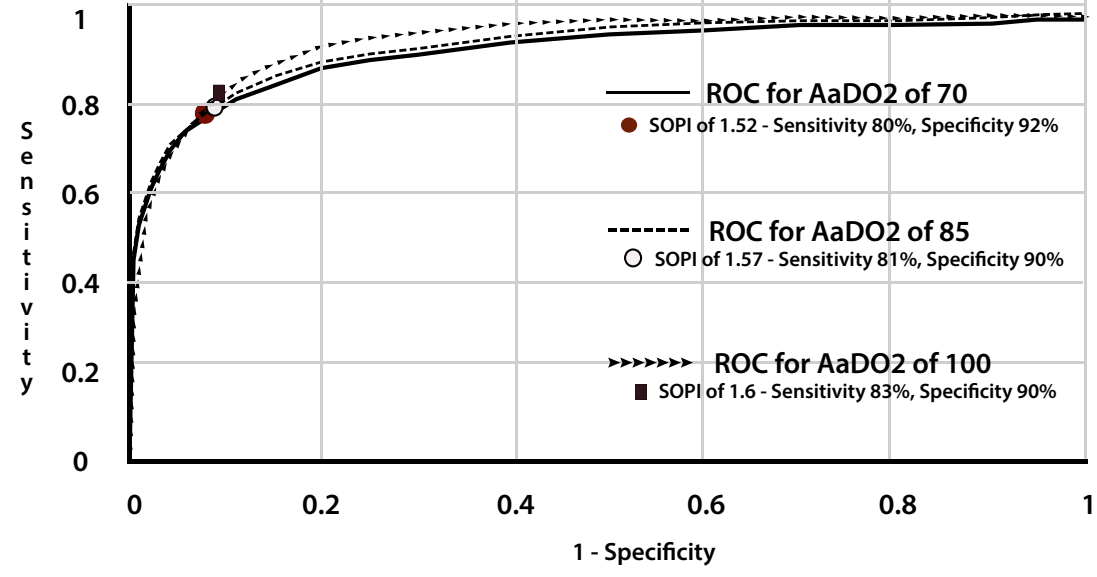

the clinician needs a score to escalate treatment rather than the other way.

Doreswamy et al. [7] in their endeavour to develop a comprehensive non-invasive tool for monitoring babies on $\mathrm{N}$ CPAP studied 36 neonates who were on mechanical ventilation. They used PEEP and FiO2 in the numerator and the outcome of treatment $\mathrm{SpO} 2$ in the denominator. Their rationale for attempting to use PEEP on ventilator babies was that in their unit, PEEP was the most commonly altered parameter to achieve alveolar recruitment. Recruiting babies on mechanical ventilation and using oxygenation index rather than $\mathrm{AaDO} 2$ was a serious limitation of that study. Nevertheless, the high correlation of SOPI with the oxygenation index proved to be a successful proof of concept.

These shortcomings were addressed in the study done by Krishnegowda et al. [8]. They recruited babies on CPAP and correlated SOPI with AaDO2. They reported a significant correlation between the two and reported a SOPI value of 1.6 to represent an $\mathrm{AaDO} 2$ of 70.

In the present study, we have incorporated two more additional aspects. One is to increase the range of $\mathrm{AaDO} 2$ to three standard deviations and obtain the SOPI value to represent the same. This will enable the clinicians to have a wider range of SOPI values to work with. The second is to calculate SOPI values with a higher specificity. Due to improved CPAP devices and understanding of managing babies on CPAP, there is a higher thrust on non-invasive management of respiratory distress. Hence, some clinicians may choose to have indicators with higher specificity before deciding on an escalation of respiratory support. SOPI values with higher specificity will increase the confidence to make major therapeutic decisions such as intubation and mechanical ventilation.

SOPI offers a near-continuous non-invasive assessment tool for babies on N-CPAP. A range of SOPI values between 1.52 and 1.88 represents various $\mathrm{AaDO} 2$ values which are of clinical importance. Each individual unit can monitor SOPI and determine an optimal threshold SOPI to take appropriate management decisions. This results in uniformity in assessment and expressing the severity of lung disease.

We did not use a chin strap or any other manoeuvres to control the leak. Open mouth can result in significant dissipation of the CPAP pressure. We made sure that the data was collected when the baby was calm and not agitated. This has limited the possibility of significant leakage influencing the results. However, an open mouth in a calm baby could have still resulted in a loss of CPAP pressure. This could be one of the minor limitations in this study. Not estimating the effect of foetal haemoglobin on oxygenation and hence saturation during the study is another limitation.

\section{Conclusion}

SOPI is an objective validated tool for non-invasive monitoring of neonates on N-CPAP. The correlation coefficient with $\mathrm{A}-\mathrm{aDO} 2$ is $82.7 \%$.

A SOPI value of $1.52,1.57$ and 1.6 indicates $\mathrm{A}-\mathrm{aDO} 2$ value of 70,85 and 100 , respectively.

A high specificity (95\%) cut-off values of SOPI $1.55,1.82$ and 1.88 indicate $\mathrm{A}-\mathrm{aDO} 2$ value of 70,85 and 100 , respectively.

Authors' contributions DT did the initial literature search, helped in study design and collected the data. She also contributed for preparation of manuscript.

AHC did the literature search, collected the data and prepared the initial manuscript.

MVG helped in study design, data analysis and preparation of the manuscript.

SMD was responsible for generating research question, study design, data analysis and interpretation. He also helped in preparing the illustration and manuscript.

Data availability Data is stored with one of the authors and the institution repository. In case of any request, authors agree to share the data under the institution (JSS Academy of Higher Education and Research, Mysuru, India) policy. 


\section{Compliance with ethical standards}

Conflict of interest All authors declare that they have no conflict of interest.

Ethics Institutional ethical board approved this study.

Consent to participate Informed verbal consent was taken from the parents or immediate caregivers for participation in this study.

Consent for publication All authors give consent to publish this article in the present form.

Code availability Not applicable.

\section{References}

1. Wiswell TE, Courtney SE (2011) Non-invasive respiratory support. In: Goldsmith JP, Karotkin EW (eds) Assisted ventilation of the neonate, 6th edn. Saunders, Philadelphia, pp 140-162

2. Shashidhar A, Suman Rao PN, Jose J (2016) Downes score vs Silverman Anderson score for assessment of respiratory distress in preterm newborns. Pediatr Oncall J 13(3):66-68. https://doi. org/10.7199/ped.oncall.2016.30

3. Dargaville PA, Aiyappan A, De Paoli AG, Dalton RGB, Kuschel CA, Kamlin CO, Orsini F, Carlin JB, Davis PG (2013) Continuous positive airway pressure failure in preterm infants: incidence, predictors and consequences. Neonatology 104(1):8-14. https://doi. org/10.1159/000346460

4. Klingenberg C, Embleton ND, Jacobs SE, O'Connell LAF, Kuschel CA (2012) Enteral feeding practices in very preterm infants: an international survey. Arch Dis Child Fetal Neonatal Ed 97(1):F56-F61. https://doi.org/10.1136/adc.2010.204123

5. Sharpe E, Kuhn L, Ratz D, Krein SL, Chopra V (2017) Neonatal peripherally inserted central catheter practices and providers: results from the neonatal PICC1 survey. Adv Neonatal Care 17(3):209 221. https://doi.org/10.1097/ANC.0000000000000376

6. Afjeh SA, Sabzehei MK, Khoshnood Shariati M, Shamshiri AR, Esmaili F (2017) Evaluation of initial respiratory support strategies in VLBW neonates with RDS. Arch Iran Med 20(3):158-164 0172003/AIM.008

7. Doreswamy SM, Chakkarapani AA, Murthy P (2015) Saturation oxygen pressure index for assessment of pulmonary disease in neonates on non-invasive ventilation. Indian Pediatr 52(1):74-75

8. Krishnegowda S, Doreswamy SM, Thandaveshwar D (2017) Comprehensive, noninvasive saturation, oxygen, and pressure index: does it reflect the severity of acute respiratory illness in neonates on continuous positive airway pressure? A prospective study. J Clin Neonatol 6(4):245. https://doi.org/10.4103/jcn.JCN 6817

9. Hiremath GM, Mukhopadhyay K, Narang A (2009) Clinical risk factors associated with extubation failure in ventilated neonates. Indian Pediatr 46(10):887-890

10. Lovering AT, Romer LM, Haverkamp HC, Pegelow DF, Hokanson JS, Eldridge MW (2008) Intrapulmonary shunting and pulmonary gas exchange during normoxic and hypoxic exercise in healthy humans. J Appl Physiol (1985) 104(5):1418-1425. https://doi.org/ 10.1152/japplphysiol.00208.2007

11. Yee K, Shetty AL, Lai K (2015) ABG needle study: a randomised control study comparing $23 \mathrm{G}$ versus $25 \mathrm{G}$ needle success and pain scores. Emerg Med J 32(5):343-347. https://doi.org/10.1136/ emermed-2014-203600

12. Soderstrom CA, Wasserman DH, Dunham CM, Caplan ES, Cowley RA (1982) Superiority of the femoral artery for monitoring: a prospective study. Am J Surg 144(3):309-312. https://doi. org/10.1016/0002-9610(82)90007-1

13. Bedford RF (1978) Wrist circumference predicts the risk of radialarterial occlusion after cannulation. Anesthesiology 48(5):377-378

14. Rawat M, Chandrasekharan PK, Williams A, Gugino S, Koenigsknecht C, Swartz D, Ma CX, Mathew B, Nair J, Lakshminrusimha S (2015) Oxygen saturation index and severity of hypoxic respiratory failure. Neonatology 107(3):161-166. https://doi.org/10.1159/000369774

15. Doreswamy SM, Chakkarapani AA, Murthy P (2016) Oxygen saturation index, a noninvasive tool for monitoring hypoxemic respiratory failure in newborns. Indian Pediatr 53(5):432-433

16. Mhanna MJ, Iyer NP, Piraino S, Jain M (2017) Respiratory severity score and extubation readiness in very low birth weight infants. Pediatr Neonatol 58(6):523-528. https://doi.org/10.1016/j.pedneo. 2016.12.006

Publisher's note Springer Nature remains neutral with regard to jurisdictional claims in published maps and institutional affiliations. 